\title{
Wakes and instabilities of static and freely vibrating cylinders
}

\author{
I. Rodríguez, O. Lehmkuhl, D. Pastrana, J.C. Cajas, and G. Houzeaux
}

\section{Introduction}

The flow around static and freely vibrating cylinders are of interest for understanding different phenomena encountered in many practical applications. In the particular case of a freely vibrating cylinder it might also be of special interest as a clean source of energy based on vortex-induced vibrations for energy harvesting in the range of frequencies where flow induced vibrations originate a strong coupling between the oscillating body and the fluctuating wake.

For the static cylinder case, the flow involves a complex physical phenomena with a strong dependency with the Reynolds number characterised by flow separations, transition to turbulence and the shedding of vortices (see for instance $[1,2,3])$. Roshko [4], based on the characteristics of the flow, classified it into different regimes: subcritical, critical, super-critical and transcritical. The subcritical regime, $R e=10^{3}-2 \times 10^{5}$, is mainly characterised by separation of the boundary layer around $\theta=90^{\circ}$ (measured from the front stagnation point), transition to turbulence in the separated shear layers and an approximately constant drag coefficient $C_{D} \approx 1$ (see figure 1). This range is also characterised by a coherent wake and an almost constant shedding of vortices at $S t=f_{v S} D / U_{\text {ref }} \approx 0.21$. The critical regime, $2 \times 10^{5}<\operatorname{Re}<5 \times 10^{5}$, is characterised by a sudden decrease in the drag coefficient accompanied by the appearance of a laminar separation bubble, first on one side of the cylinder which produces a positive lift coefficient, and then on both sides of the

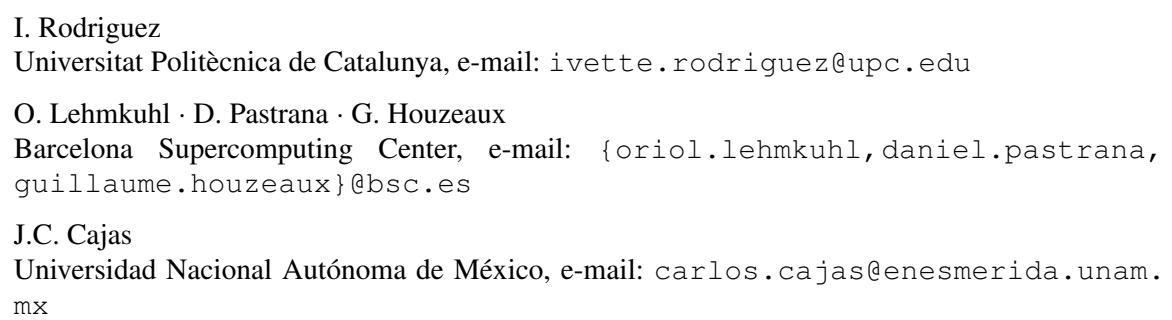

Rodríguez, I. [et al.]. Wakes and instabilities of static and freely vibrating cylinders. A: ERCOFTAC Workshop on Direct and Large-Eddy Simulation. "Direct and Large Eddy Simulation XII". Berlín: Springer, 2019, p. 49-59. The final authenticated version is available online at https://doi.org/10.1007/978-3-030-42822-8_7 
cylinder $\left([5,6,7]\right.$. At $R e>5 \times 10^{5}$, the flow enters the super-critical regime with the drag coefficient reaching its minimum value of $C_{D} \approx 0.2$. Traditionally, the supercritical wake has been described as incoherent $[4,8,1,3,9]$, with turbulent transition at separation. However, different authors have measured coherent vortex shedding in this regime (see for instance the experimental works of $[10,5,11]$ ), and as was pointed out in Lehmkuhl et al. [7] and Rodriguez et al. [12], vortex shedding was detected at super-critical Reynolds numbers between $R e=6.5 \times 10^{5}-8.5 \times 10^{5}$. In these two studies, the wake has been described as a coherent vortex street more compact than that observed at subcritical Reynolds numbers (see figure 2). The nondimensional vortex shedding frequency measured in the super-critical regime was around $S t=f_{v s} D / U_{r e f}=0.45$ in agreement with experimental measurements of $[5,11]$.

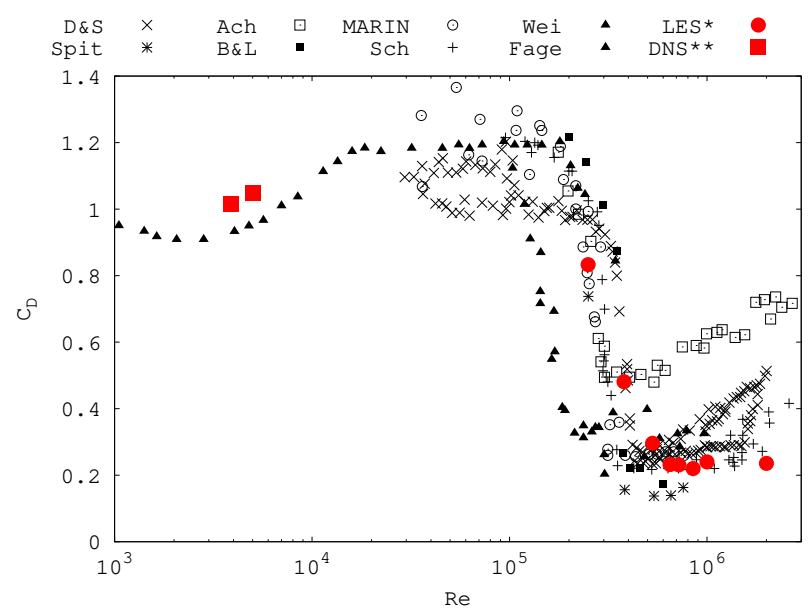

Fig. 1 Drag coefficient in cylinders from subcritical to super-critical regime. Comparison with the literature. (red solid circles) DNS [13, 14] and LES [7, 12]; (D\&S) [15]; (Spit) [16]; (Ach) [6]; (B\&L) [17]: (MARIN) [18]; (Sch) [11]; (Wei) [19]; (Fage) [20]

All these features get more complicated when the cylinder is freely to vibrate in two-degrees of freedom (2dof), as the wake characteristics are not only affected by the Reynolds number, i.e. whether the flow is in the subcritical regime or in the super-critical regime, but also by the structural response of the system as a function of the reduced velocity $U^{*}=U_{\infty} /\left(f_{n} D\right)$ ( i.e. the inverse of the natural frequency) non-dimensionalised with the free stream velocity $U_{\infty}$ and the diameter of the cylinder $D$ (see for instance the reviews of $[21,22,23,24]$ ). In this contribution, some characteristics of the wake and instabilities of a 2 dof freely vibrating cylinder are described. To do this, results from large-eddy simulations of the flow performed at different Reynolds numbers and reduced velocities are used. 


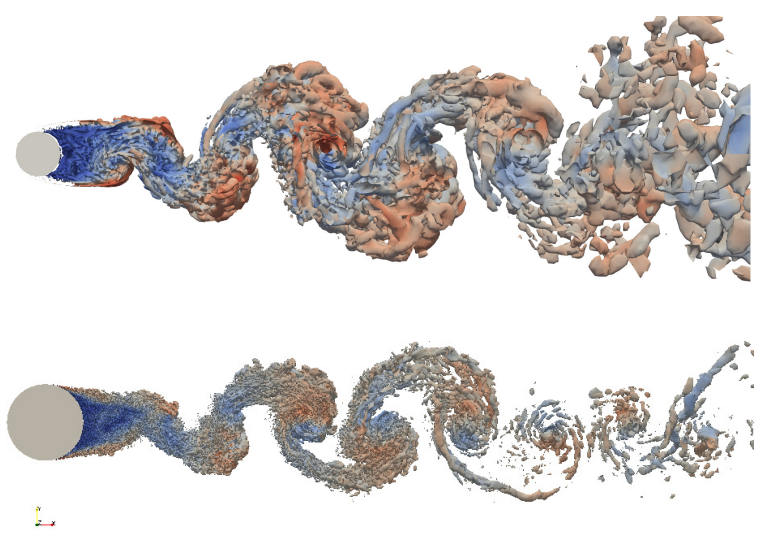

Fig. 2 The wake of a static circular cylinder (top) subcritical regime $R e=3900$, (bottom) supercritical regime $\operatorname{Re}=7.2 \times 10^{5}$.

\section{Relevant results}

The system under consideration consists of a rigid cylinder elastically mounted with springs that allow the cylinder to move in the in-line and cross-flow directions. The problem is governed by the Reynolds number together with three other parameters which characterise the structure: the fluid-structure mass ratio, the reduced velocity and the damping ratio,

$$
m^{*}=4 m /\left(\rho_{f} \pi D^{2}\right) ; \quad U^{*}=U_{\infty} /\left(f_{n} D\right) ; \quad \zeta=c /(2 \sqrt{k m})
$$

where $m$ is the mass of the cylinder, $\rho_{f}$ the density of the fluid and $f_{n}$ the natural frequency of the body $f_{n}=(2 \pi)^{-1} \sqrt{k / m}$ with $k$ the structural stiffness and $c$ is the structural damping. The body vibrations are driven by the transient fluid forces. The in-line and cross-flow forces coefficients are defined as $\mathbf{C}=2 \mathbf{F} /\left(\rho_{f} D U_{\infty}^{2}\right)$, $\mathbf{C} \equiv\left(C_{x}, C_{y}\right)$ and $\mathbf{F} \equiv\left(F_{x}, F_{y}\right)$ being the in-line and cross-flow sectional forces. The system is solved using the code Alya [25]. The low-dissipation methodology for solving the flow is described in detail in Lehmkuhl et al. [26] and the methodology used for the resolution of the fluid-structure interaction system can be found in Cajas et al. [27] and Pastrana et al. [28].

The most relevant features regarding the wake configuration and instabilities that appears in 2dof cylinders are hereafter commented. For the analysis, large-eddy simulations at Reynolds numbers in the range of $R e=3900-7.5 \times 10^{5}$ are considered. Notice that this range covers both the subcritical and super-critical regimes. In order to analyse the response of the system, reduced velocities range is $U^{*}=3.0-12$ for the subcritical regime and $U^{*}=0.5-5.0$ for the super-critical regime. In all simulations, a low mass ratio $m^{*}=2.6$ and zero damping $\zeta=0$ system is considered. Moreover, in this work the structural stiffness and damping are equal in both directions $\left(k_{x}=k_{y}=k\right.$ and $\left.c_{x}=c_{y}=c\right)$. 


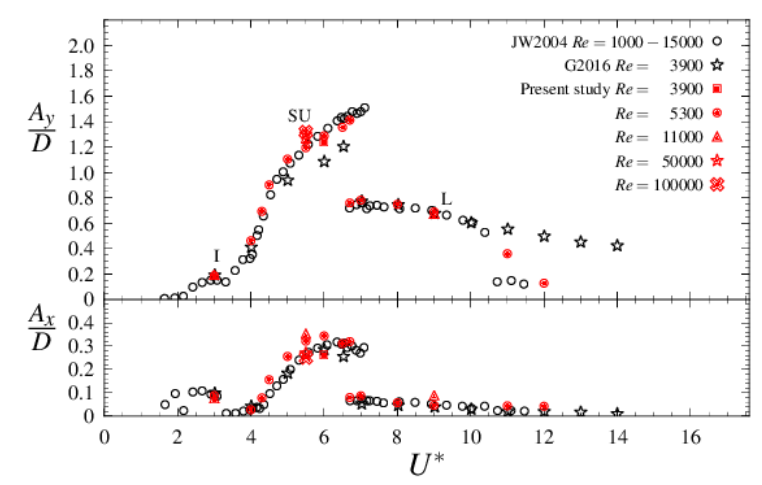

Fig. 3 Non-dimensional cross-flow and in-line maximum amplitudes of oscillation as a function of the reduced velocity. Present LES results at different Reynolds numbers compared with the experimental data of JW04 and the computational data of G10.

\section{The subcritical wake}

In figure 3, the maximum cross-flow and in-line non-dimensional amplitudes of vibration for different Reynolds numbers up to $R e=1 \times 10^{5}$, corresponding to the subcritical regime, are plotted. In the figure, the experimental results of Jauvtis \& Williamson (JW04) [29] for a low-mass ratio cylinder $m^{*}=2.6$ and in the range of $R e=1000-15000$ are given. In addition, the numerical results by Gsell et al. (G10) [30] at a Reynolds number of $R e=3900$ are also included. At these Reynolds numbers, the low-mass ratio 2 dof cylinder response is characterised by three branches: the initial $(I)$, the super-upper $(S U)$ and the lower $(L)$ branches. Each of these branches have different characteristics and amplitude of response.

The $I$ branch, where the synchronisation or lock-in begins, has very small in-line vibrations (see figure 3) and the wake is characterised by the typical von Kármán street vortex shedding mode or $2 \mathrm{~S}$ vortex formation mode (following the nomenclature of Williamson \& Roshko [31]), i.e. 2 vortices shed each cycle. This wake configuration can be seen in figure 4 (top), where ensemble average vorticity contours at $U^{*}=3.0$ are depicted. Actually, in the $2 \mathrm{~S}$ mode, and in spite of the cross-flow fluctuation of the cylinder, the vortices shed are in-line with the wake centreline in a similar fashion to the static cylinder as can be seen in the figure.

Of particular interest is the $S U$ branch, where in-line and cross-flow amplitudes of fluctuation peak. This branch is not present in 1dof systems and is characteristic only of 2dof with low-mass ratio $m *<6$ [29]. In this branch, the wake is broader (see figure 4 (middle)) and vortex shedding mode changes from a $2 \mathrm{~S}$ pattern to a $2 \mathrm{~T}$ one, i.e. 2 triplets of vortices shed each shedding cycle. This mode was first described by JW04. Actually in figure 4, the footprint of two out of three vortices shed once the cylinder reaches its maximum elongation in the cross-flow direction is observed. However, as the flow moves downstream, the triplets of vortices shed each 

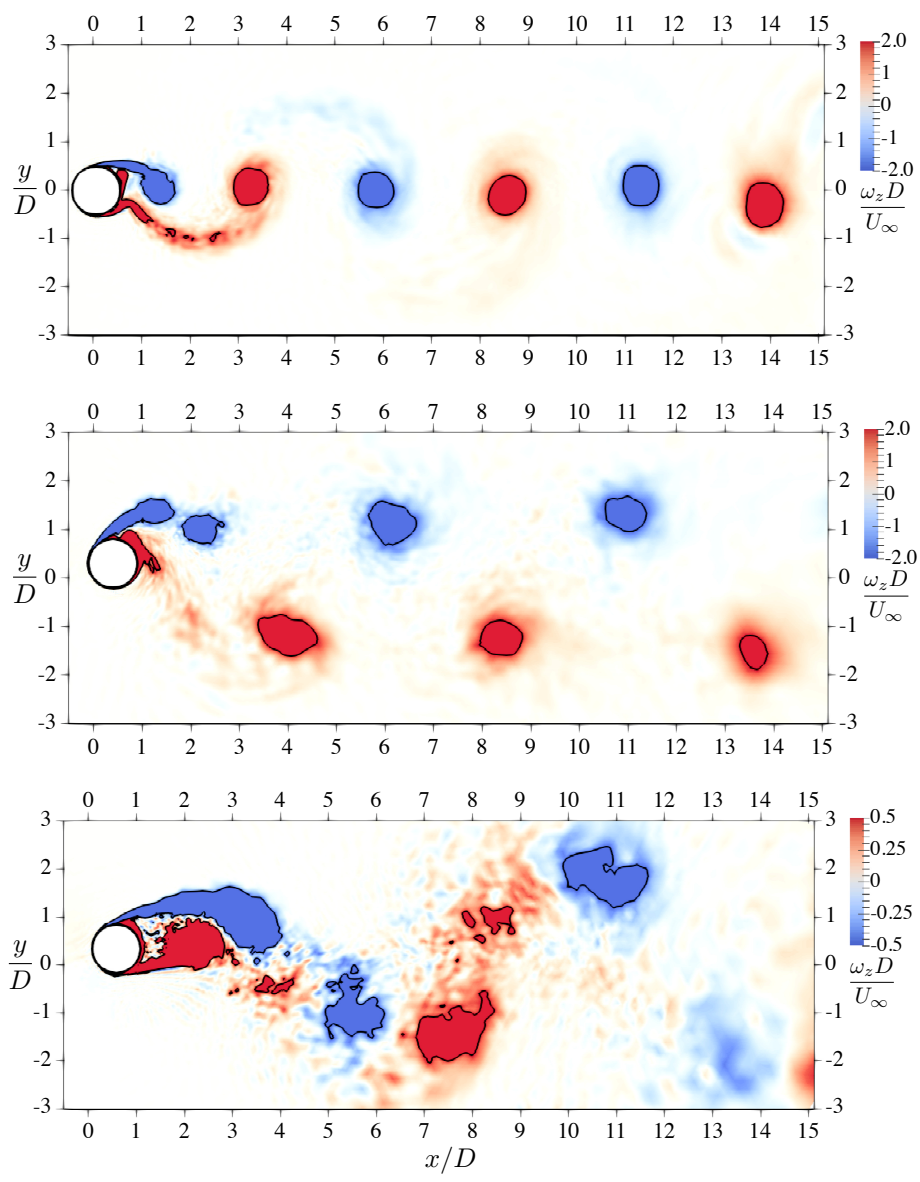

Fig. 4 The 2dof freely vibrating cylinder wake. Ensemble average vorticity contours in the initial branch $U^{*}=3.0$ (top), the super-upper branch $U^{*}=5.5$ (middle) and, the lower branch $U^{*}=9.0$ (bottom).

cycle merge into a single vortex located off the wake centreline. Finally, the $L$ branch (see figure 4 (bottom)), with a low level of fluctuation in the in-line direction and with decreasing cross-flow amplitudes as the reduced velocity increases up until the de-synchronisation of the system around $U^{*}=12$ occurs. In this branch the vortex shedding mode is different than in the other two branches and is characterised by two pair of vortices shed each cycle (also known as $2 \mathrm{P}$ mode), as it can be seen in figure 4 (bottom).

Last but not the least, attention should be also paid to the fact that along the whole subcritical regime, cylinder amplitudes are only function of the reduced velocity and independent on the Reynolds number (see figure 3). In fact, the cylinder in-line and cross-flow positions at each instant of the the cycle(not shown here) are almost the same regardless of the Reynolds number, which points out to the similarities with the 
static cylinder in which along the whole subcritical regime the wake characteristics are nearly the same. Actually, if the instantaneous flow at $R e=5300$ and $R e=10^{5}$ for $U^{*}=5.5$ are compared at the maximum elongation position, one can see the same large scale structures of the flow and wake configuration for both Reynolds numbers (see figure 5).
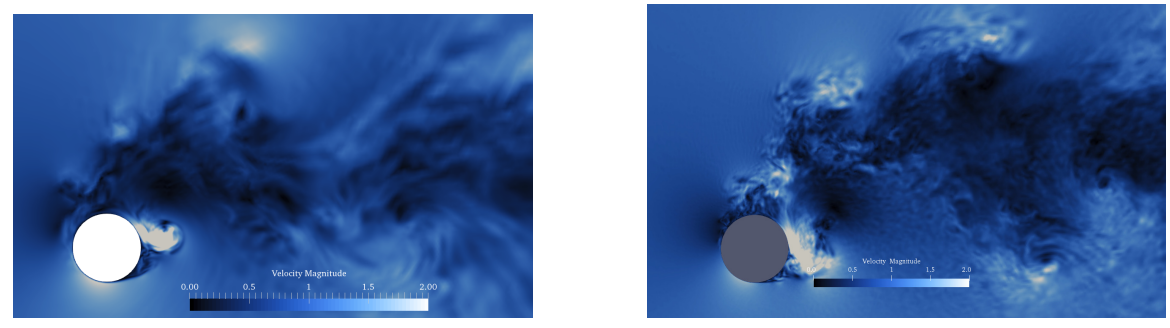

Fig. 5 Instantaneous flow in the subcritical regime in the super-upper branch at $U^{*}=5.5 . R e=$ 5300 (left) and $\operatorname{Re}=10^{5}$ (right).

\section{The super-critical wake}

In figure 6, the instantaneous flow at $R e=7.5 \times 10^{5}$ at $U^{*}=2.5$ is depicted together with the non-dimensional cross-flow and in-line amplitudes of fluctuation as a function of the reduced velocity. Similar to the static cylinder, the super-critical wake of the 2dof vibrating cylinder is narrower than in the subcritical regime. In a similar manner, the boundary layer separates past the cylinder apex, on both sides of the cylinder. As a result, the separated shear layers are closer each other and thus the fluctuations produced due to their interaction are also small. As a consequence of this super-critical flow configuration, synchronisation and de-synchronisation are attained at lower reduced velocities than in the subcritical regime, as can also be seen in figure 6 (right). Although the range of reduced velocities covered by the present study is quite discrete, it seems that in the super-critical regime only initial and lower branches are present, with a behaviour (in terms of the amplitude of fluctuations which are almost negligible in the in-line direction) similar to that observed in high-mass ratio 1dof cylinders (see for instance [32]).

\section{Instabilities in the cylinder surface}

Similar to the differences observed in the wake of the cylinder, three-dimensional structures triggered and developed are also different depending on the branch of response (see figure 7). However, both initial and lower branches present some sim- 

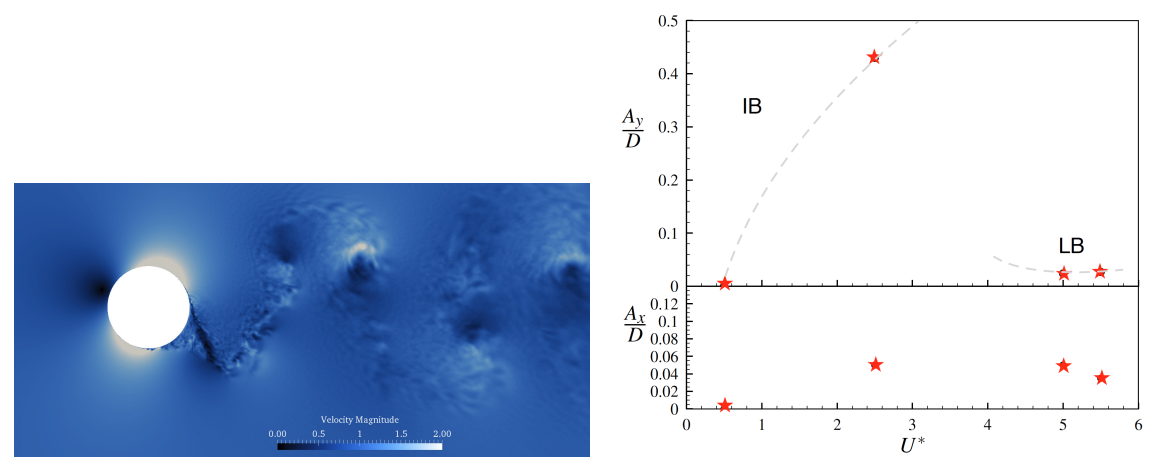

Fig. 6 The super-critical wake of a 2 dof cylinder at $R e=7.5 \times 10^{5}$ (right) and non-dimensional cross-flow and in-line amplitudes as a function of the reduced velocity in the super-critical regime.

ilarities. In these two branches, three-dimensional instabilities are triggered in the separated shear layers the boundary layer being laminar during the whole fluctuation cycle. Nonetheless, a noticeable difference between these two branches is related with the length of the vortex formation zone. While in the former, shear layers roll-up close to the cylinder surface, in the latter both shear layers are almost in phase with little interaction between each other. As a consequence, the inception of instabilities that the vortex induced vibration (VIV) produces occurs farther from the cylinder and thus, vortex formation zone is larger than in the initial branch. A fundamental change is produced in the super-upper branch, where the oscillation of the cylinder and the increase in the local angular velocity trigger a centrifugal instability in the boundary layer, forming Görtler-like structures. These structures form and dissipate periodically twice every cycle depending on the position of the cylinder (see also figure 8) and can be seen as counter-rotating vortical structures covering the whole span. Similar structures have been observed before in cylinders with forced oscillations in a fluid at rest [33,34], in the flow over a rotating cylinder [35] and in the flow over a cylinder performing rotating oscillations [36], but have never been reported on the VIV context.

The spanwise length of the Görtler-like structures can be determined by applying the Hilbert transform to the signal of probes located in lines parallel to the cylinder axis in the boundary layer. The local wavelength of the signal $w(z)$ is obtained, $k D=\frac{\mathrm{d} \phi}{\mathrm{d} z}, \phi$ being its local amplitude. Thus, the spanwise length is defined as $\lambda_{z} / D=2 \pi /(k D)$. The mean value $\overline{\lambda_{z}} / D$ is determined as the temporal average of the mean of the $5 \%$ most frequent wavelengths. These structures are observed in the super-upper branch, in this work, for reduced velocities $U^{*}=4.3,5.5,6.5$; the average spanwise length is $\overline{\lambda_{z}} / D=0.280,0.322$ and 0.311 , respectively. For the sinusoidal oscillatory flow around a circular cylinder, Sarpkaya [34] using data from experimental measurements obtained the correlation $\lambda_{z} / D=22 \beta^{-3 / 5}, \beta=f D^{2} / v$ being the Stokes number. Using the correlation, the size of the structures obtained is $\lambda_{z} / D=0.255,0.284,0.276$, which is comparable to the values measured in the 
freely oscillating cylinder. Thus, it can be stated that in 2 dof freely vibrating cylinders, when the cylinder reaches its maximum amplitudes of fluctuation (i.e. in the $S U$ branch), centrifugal instabilities might trigger three-dimensional structures in the boundary layer. These structures have similar characteristics to those observed in systems such as forced sinusoidal oscillating cylinders.
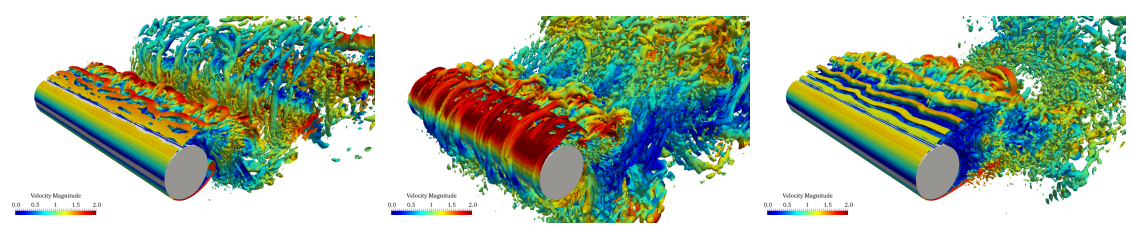

Fig. 7 Instantaneous vortical structures close to the cylinder surface. Initial branch (left), superupper branch (middle), lower branch (right).
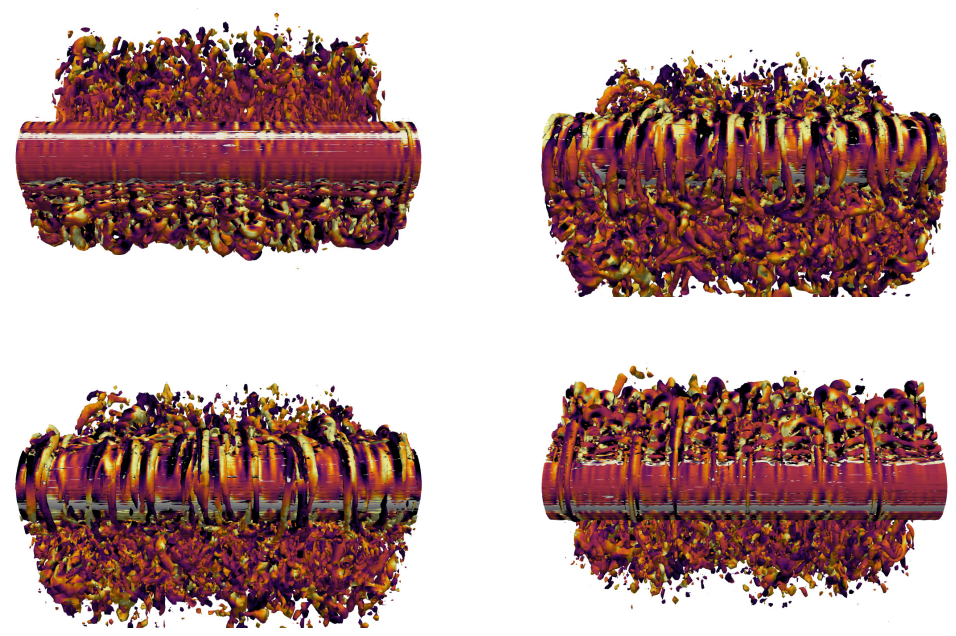

Fig. 8 Görtler-like structures observed in the super-upper branch. The image represents different positions of the cylinder during one cycle. The Görtler-like structures are observed (top right and bottom left) in the zone where the cylinder attains its maximum elongation.

\section{Summary}

The main characteristics of the wake of 2 dof freely vibrating cylinders are described using results from large-eddy simulations of the flow in the range of Reynolds numbers of $R e=3900-7.5 \times 10^{5}$, which covers both the subcritical and the super- 
critical regimes. The behaviour of 2 dof vibrating cylinders remains almost the same along the whole subcritical regime $\left(R e=3900-10^{5}\right)$ and the three-branches of response of this system is well captured by the LES. In the super-critical regime, the wake is narrower than that observed in the subcritical regime and only two branches of response ( $I$ and $L$ ) are observed, with in-line fluctuations almost negligible and a behaviour similar to that observed in high-mass ratio 1 dof systems. In the $S U$ branch a centrifugal instability appears and as a consequence, Görtler-like structures form in the boundary layer twice every cycle; the size of these structures being comparable to the values measured in forced oscillating cylinders in a fluid at rest.

Acknowledgements This work has been partially financially supported by the Ministerio de Economía y Competitividad, Secretaría de Estado de Investigación, Desarrollo e Innovación, Spain (Ref. TRA2017-88508-R). We also acknowledge Red Española de Surpercomputación (RES) for awarding us access to the MareNostrum IV machine based in Barcelona, Spain (Ref. FI-2017-20016) and PRACE for awarding us access to SuperMUC (Project ViValdi Ref. 2017174222). D. Pastrana acknowledges support of the CONACyT-SENER graduate fellowship program to study abroad 278102/439162.

\section{References}

1. A. Roshko, "Perspectives on bluff body aerodynamics," J. Wind Eng. and Aerod., vol. 49, no. 1, pp. 79-100, 1993.

2. C. H. K. Williamson, "Vortex dynamics in the cylinder wake," Annual Review of Fluid Mechanics, vol. 28, no. 1, pp. 477-539, 1996.

3. M. Zdravkovich, "Flow around circular cylinders. Volume I: Fundamental," J. Fluid Mech., vol. 350, no. 1, pp. 375-378, 1997.

4. A. Roshko, "Experiments on the flow past a circular cylinder at very high Reynolds number," Journal of Fluid Mechanics, vol. 10, no. 3, pp. 345-356, 1961.

5. P. W. Bearman, "On vortex shedding from a circular cylinder in the critical Reynolds number regime," J. Fluid Mech, vol. 37, pp. 577-585, 1969.

6. E. Achenbach and E. Heinecke, "On vortex shedding from smooth and rough cylinders in the range of Reynolds numbers 6e3 to 5e6," J. Fluid Mech, vol. 109, pp. 239-251, 1981.

7. O. Lehmkuhl, I. Rodríguez, R. Borrell, J. Chiva, and A. Oliva, "Unsteady forces on a circular cylinder at critical Reynolds numbers," Physics of Fluids, vol. 26, no. 12, p. 125110, 2014.

8. J. Lienhard, "Synopsis of Lift, drag and cortex shedding frequency data fro rigid circular cylinders," Tech. Rep. Bulletin300, College of Engineering. Research Division, 1966.

9. W. Shih, C. Wang, D. Coles, and A. Roshko, "Experiments on flow past rough circular cylinders at large Reynolds numbers," Journal of Wind Engineering and Industrial Aerodynamics, vol. 49, pp. 351-368, 1993.

10. E. Achenbach, "Distribution of local pressure and skin friction around a circular cylinder in cross-flow up to Re=5e6," J. Fluid Mech, vol. 34, pp. 625-639, 1968.

11. G. Schewe, "On the force fluctuations acting on a circular cylinder in crossflow from subcritical up to transcritical Reynolds numbers," Journal of Fluid Mechanics, vol. 133, pp. 265-285, 1983.

12. I. Rodríguez, O. Lehmkuhl, J. Chiva, R. Borrell, and A. Oliva, "On the flow past a circular cylinder from critical to super-critical Reynolds numbers: Wake topology and vortex shedding," International Journal of Heat and Fluid Flow, vol. 55, pp. 91-103, 2015.

13. O. Lehmkuhl, I. Rodríguez, R. Borrell, and A. Oliva, "Low-frequency unsteadiness in the vortex formation region of a circular cylinder," Physics of Fluids, vol. 25, p. 085109, 2013. 
14. D. Aljure, O. Lehmkhul, I. Rodríguez, and A. Oliva, "Three dimensionality in the wake of the flow around a circular cylinder at Reynolds number 5000," Computers \& Fluids, vol. 147, pp. 102-118, 2017.

15. N. Delany and N. Sorensen, "Low-speed drag of cylinders of various shapes," tech. rep., NACA, 1953.

16. R. Spitzer, Measurements of unsteady pressures and wake fluctuations for flow over a cylinder at supercritical Reynolds number. PhD thesis, California Institute of Technology, 1965.

17. W. Bursnall and L. J. Loftin, "Experimental investigation of the pressure distribution about a yawed circular cylinder in the critical Reynolds number range," tech. rep., NACA, 1951.

18. G. Vaz, C. Mabilat, R. van der Wal, and P. Gallagher, "Viscous flow computations on smooth cylinders: A detailed numerical study with validation," in 26th International Conference on Offshore Mechanics and Artic Engineering. OMAE2007, (San Diego, California), 2007.

19. C. Wieselsberger, "New data on the laws of fluid resistance," Tech. Rep. TN 84, NACA, 1921.

20. A. Fage, "Drag of circular cylinders and spheres," tech. rep., Aeronautical Research Council, 1930.

21. T. Sarpkaya, "Vortex-induced oscillations: A selective review," Journal of Applied Mechanics, vol. 46, pp. 241-258, 1979.

22. T. Sarpkaya, "A critical review of the intrinsic nature of vortex-induced vibrations," Journal of Fluids and Structures, vol. 19, no. 4, pp. 389-447, 2004.

23. C. H. K. Williamson and R. Govardhan, "A brief review of recent results in vortex-induced vibrations," Journal of Wind Engineering and Industrial Aerodynamics, vol. 96, pp. 713-735, 2008.

24. P. W. Bearman, "Circular cylinder wakes and vortex-induced vibrations," Journal of Fluids and Structures, vol. 27, no. 5-6, pp. 648-658, 2011.

25. M. Vázquez, G. Houzeaux, S. Koric, A. Artigues, J. Aguado-Sierra, R. Arís, D. Mira, H. Calmet, F. Cucchietti, H. Owen, A. Taha, E. D. Burness, J. M. Cela, and M. Valero, "Alya: Multiphysics engineering simulation towards exascale," J. Comput. Sci., vol. 14, pp. 15-27, 2016.

26. O. Lehmkuhl, G. Houzeaux, H. Owen, G. Chrysokentis, and I. Rodriguez, "A low-dissipation finite element scheme for scale resolving simulations of turbulent flows," Journal of Computational Physics, vol. 390, pp. 51-65, 2019.

27. J. Cajas, G. Houzeaux, M. Vázquez, M. García, E. Casoni, H. Calmet, A. Artigues, R. Borrell, O. Lehmkuhl, D. Pastrana, D. Yáñez, R. Pons, and J. Martorell, "Fluid-structure interaction based on HPC multicode coupling," SIAM J. Sci. Comput.,, vol. 40, no. 6, pp. C677-C703, 2018.

28. D. Pastrana, J. C. Cajas, O. Lehmkuhl, I. Rodríguez, and G. Houzeaux, "Large-eddy simulations of the vortex-induced vibration of a low mass ratio two-degree-of-freedom circular cylinder at subcritical Reynolds numbers," Comput. Fluids., vol. 173, pp. 118-132, 2018.

29. N. Jauvtis and C. H. K. Williamson, "The effect of two degrees of freedom on vortex-induced vibration at low mass and damping," J. Fluid Mech., vol. 509, pp. 23-62, 2004.

30. S. Gsell, R. Bourguet, and M. Braza, "Two-degree-of-freedom vortex-induced vibrations of a circular cylinder at Re=3900," J. Fluids Struct., vol. 67, pp. 156-172, 2016.

31. C. H. K. Williamson and A. Roshko, "Vortex formation in the wake of an oscillating cylinder," J. Fluids Struct., vol. 2, no. 4, pp. 355-381, 1988.

32. C. Williamson and R. Govardhan, "Vortex-induced vibrations," Annu. Rev. Fluid Mech., vol. 36, pp. 413-455, 2004.

33. H. Honji, "Streaked flow around an oscillating circular cylinder," J. Fluid Mech., vol. 107, p. 509-520, 1981.

34. T. Sarpkaya, "Experiments on the stability of sinusoidal flow over a circular cylinder," J. Fluid Mech., vol. 457, p. 157-180, 2002.

35. D. E. Aljure, I. Rodríguez, O. Lehmkuhl, C. D. Pérez-Segarra, and A. Oliva, "Influence of rotation on the flow over a cylinder at $\mathrm{Re}=5000$," International Journal of Heat and Fluid Flow, vol. 55, pp. 76-90, 2015.

36. J. D'Adamo, R. Godoy-Diana, and J. E. Wesfreid, "Centrifugal instability of Stokes layers in crossflow: the case of a forced cylinder wake," Proc. R. Soc. Lond. A Math. Phys. Sci., vol. 471, no. 2178, p. 20150011, 2015. 